\title{
A torus theorem for homotopy nilpotent loop spaces
}

\author{
Cristina Costoya, Jérôme Scherer and Antonio Viruel
}

\begin{abstract}
Nilpotency for discrete groups can be defined in terms of central extensions. In this paper, the analogous definition for spaces is stated in terms of principal fibrations having infinite loop spaces as fibers, yielding a new invariant between the classical LS cocategory and the more recent notion of homotopy nilpotency introduced by Biedermann and Dwyer. This allows us to characterize finite homotopy nilpotent loop spaces in the spirit of Hubbuck's Torus Theorem, and obtain corresponding results for $p$-compact groups and $p$-Noetherian groups.
\end{abstract}

\section{Introduction}

Hubbuck's Torus Theorem, [27, Theorem 1.1], characterizes, up to homotopy, classical homotopy commutative finite $H$-spaces as tori, i.e. finite products of circles. Mod $p$ versions were established after by Lin [32, Theorem 1] and Aguadé-Smith [2, Corollary], and many extensions of this theorem have been obtained by relaxing the finiteness conditions. For example, Castellana-Crespo-Scherer [12, Corollary 7.4] proved that connected homotopy commutative $H$-spaces with finitely generated cohomology as algebra over the Steenrod algebra, at the prime 2, are homotopically equivalent to products of a torus and a Potsnikov piece. In this paper we relax instead the commutativity assumption by considering the Biedermann-Dwyer

The authors are supported by Xunta de Galicia grant EM2013/016. The first author is supported by Ministerio de Economía y Competitividad (Spain), grant MTM2016-79661-P (AEI/FEDER, UE, support included). The second author is supported by Ministerio de Economía y Competitividad (Spain), grant MTM2016-80439-P. The third author is supported by Ministerio de Economía y Competitividad (Spain), grants MTM2013-41768-P and MTM2016-78647-P (AEI/FEDER, UE, support included).

Key words and phrases: nilpotent, homotopy nilpotent, cocategory, algebraic theory, Goodwillie calculus, excisive functor, $p$-compact group.

2010 Mathematics Subject Classification: primary 55P35; secondary 55P65, 18C10, 55M30. 
notion of homotopy nilpotent loop spaces and show that tori are the only homotopy nilpotent finite loop spaces.

Biedermann and Dwyer, [10, Definition 5.4], used the stages of the Goodwillie tower of the identity to provide the first definition of homotopy nilpotency which interpolates between infinite loop spaces (homotopy commutative groups or, equivalently, homotopy 1-nilpotent groups) and loop spaces. Even though this might come as a surprise to those which are more accustomed to relate the Goodwillie tower of the identity to $v_{n}$-periodicity, as discovered by Arone and Mahowald, [5], there is a quite straightforward relationship between the essence of Goodwillie calculus, namely higher excision, and nilpotency.

In order to explain this let us first go back to group theoretical nilpotency. The nilpotency of a discrete group is understood either as the minimal number of iterated commutators that must always vanish, or as the minimal number of central extensions needed to construct the group. The commutators approach has been successfully interpreted in homotopy theory leading to the classical Berstein-Ganea nilpotency [8]. Work of Hopkins, [24], and Rao, [40], gives a complete understanding of the classical Berstein-Ganea nilpotency for compact Lie groups: those with finite nilpotency index are precisely the torsion free ones. This is the case of the 3-dimensional sphere, an example which has been studied by Porter in the early 1960s, [39].

The central extensions approach will be this article's viewpoint. In homotopy theory a commutative (or 1-nilpotent) group is an infinite loop space as it should be commutative not only up to homotopy, but up to all higher homotopies. Therefore a natural analogue notion of nilpotency for loop spaces (i.e. group like spaces) is the following formal translation. We replace central extensions by principal fibrations whose fiber is an infinite loop space and, the invariant which is introduced in this way is the minimal number of extensions by such principal fibrations needed to construct a given loop space. We call this invariant the extension by principal fibrations length, or epfl for short. Let us mention that considering a central extension of groups as a principal fibration by taking classifying spaces, is a classical procedure (see [1, Lemma IV.1.12]). It appears notably in algebraic K-theory, in Quillen's use of the plus construction, a point of view adopted for example in [7].

The relationship to Goodwillie calculus is provided by the structure of the Goodwillie tower. Let $F$ be a functor from spaces to spaces and $F \rightarrow P_{n} F$ denote the $n$-excisive approximation of $F,[21$, Section 1$]$. This means that $P_{1} F$ is basically a homological functor turning homotopy push-outs into homotopy pull-backs and more generally $P_{n} F$ satisfies higher excision, [20, Definition 2.1]. The homotopy fiber $D_{n} F$ of the natural transformation $P_{n} F \rightarrow P_{n-1} F$ is a homogeneous $n$-excisive functor, [21, Proposition 1.17], and Goodwillie showed that it is classified by a spec- 
trum together with an action of the symmetric group $\Sigma_{n}$, [21, Section 5]. Concretely $D_{n} F(X)$ is the infinite loop space corresponding to the $\Sigma_{n}$-equivariant smash product of this spectrum with $X^{\wedge n}$. Even better, this fibration of functors is actually principal, [21, Lemma 2.2].

In this paper, we prove the following.

Theorem 1.5. Let $Z$ be a pointed connected space. Then, we have the inequalities

$$
\operatorname{nil}_{\mathrm{BG}}(\Omega Z) \leq \operatorname{cocat}(Z) \leq \operatorname{epfl}(Z) \leq \operatorname{nil}_{\mathrm{BD}}(\Omega Z) .
$$

Here the two nilpotency invariants refer to the classical Berstein-Ganea nilpotency, nil ${ }_{\mathrm{BG}}$ (see Section 1.2), and the Biedermann-Dwyer nilpotency, nil $\mathrm{BD}$ (see Section 1.1).

We mention finally two problems where our epfl invariant is key to the solution. First we recover the vanishing of iterated Whitehead products in values of excisive functors. If $F$ is an $n$-excisive functor from the category of pointed spaces to pointed spaces and $K$ a finite space, then $\Omega F(K)$ is a homotopy nilpotent group of class $n$, [10, Corollary 9.3]. Thus Theorem 1.5 immediately implies that all $(n+1)$-fold iterated Whitehead products vanish in $F(K)$. This has been proven originally in [14] relying on Goodwillie's generalized Blakers-Massey Theorem and the definition itself of an $n$-excisive functor. In [18] Eldred obtains another proof by analyzing Goodwillie's construction of the $n$-excisive approximation $P_{n} F$ of a functor $F$. She shows in fact that Whitehead products already vanish in $T_{n} F$, a functor directly related to Hopkins' symmetric cocategory, [25, Definition p. 219].

The second problem, from which the title of this paper comes from, concerns our understanding of finite homotopy nilpotent loop spaces, with which we come back to the first lines of this introduction. We recall briefly that a loop space $(X, B X)$ consist of a pair of pointed spaces $X$ and $B X$, together with a homotopy equivalence $e: \Omega B X \simeq X$ defining a loop structure on $X$. The space $B X$ is called the classifying space of $X$ and $(X, B X)$ is said to be finite if $H^{*}(X ; \mathbb{Z})$ is finitely generated as a graded abelian group. In this paper we offer the following version of Hubbuck's Torus Theorem for finite loop spaces. Whereas the original statement, [27, Theorem 1.1], focuses on homotopy commutative $H$-spaces, we deal with arbitrary homotopy nilpotent groups.

Theorem 3.6. Let $(X, B X)$ be a connected finite loop space. If $\operatorname{nil}_{\mathrm{BD}}(X)$ is finite, then $X$ has the homotopy type of a torus.

We obtain analogous characterizations of Biedermann-Dwyer nilpotency for $p$-compact groups (Theorem 3.1) and $p$-Noetherian groups (Corollary 3.5). Two key ingredients in the proof are, on the one hand, the existence of a finite tower 
of principal fibrations and, on the other hand, the effect of Neisendorfer's functor, [38], on the fibers of this tower making them contractible (see Section 2).

The result above shows that, in particular, simple compact Lie groups are not nilpotent loop spaces in the sense of Bierdermann-Dwyer, in contrast to the classical situation when considering Berstein-Ganea nilpotency where all torsion free simple Lie groups are nilpotent [25] and [40]. This provides yet more evidence that nil $\mathrm{BD}$ is the correct notion for nilpotency of loop spaces.

Acknowledgments. The first and third author would like to acknowledge the hospitality of the EPFL, where this paper has been completed. We would like to thank Georg Biedermann for sharing his unpublished results with us, and the referees for their careful reading and constructive comments.

\section{Background and first results}

In this section we briefly introduce the main ingredients in Theorem 1.5.

\subsection{Nilpotency in the sense of Biedermann-Dwyer}

We need some basic notions on algebraic theories to understand the nilpotency in the sense of Biedermann-Dwyer. Algebraic theories were introduced by Lawvere [31] to describe algebraic structures, and successfully interpreted in homotopy theory by Badzioch [6]. We will need simplicial theories and follow closely the viewpoint from [10, Section 3].

Definition 1.1. An algebraic theory is a small category $T$ whose objects are indexed by the natural numbers $\left\{T_{0}, T_{1}, \ldots, T_{n}, \ldots\right\}$ such that for $n \in \mathbb{N}$ the $n$-fold categorical coproduct of $T_{1}$ is naturally isomorphic to $T_{n}$.

In our situation, the algebraic theory $T$ will be required to be pointed and simplicial, which means that $T$ is enriched over the category $\mathcal{S}_{*}$ of pointed simplicial sets. We distinguish between strict and homotopy $T$-algebras: simplicial functors $\underset{\sim}{X}: T^{\mathrm{op}} \rightarrow \mathcal{S}_{*}$ taking coproducts in $T^{\mathrm{op}}$ to products in $\mathcal{S}_{*}$ strictly or up to homotopy, respectively.

Biedermann and Dwyer define homotopy nilpotent groups as homotopy $\mathcal{G}_{n}$-algebras in the category of pointed spaces, where $\mathcal{G}_{n}$ is a simplicial algebraic theory constructed from the Goodwillie tower of the identity, [10, Definition 5.4]. Concretely, $\mathcal{G}_{n}^{\text {op }}$ is the simplicial category which has for each natural number $k \geq 0$ exactly one object given by $\mathcal{G}_{n}(k)=\prod_{k} \Omega\left(P_{n}(\mathrm{id})\right)^{\mathrm{inj}}$. The functor $P_{n}(\mathrm{id})$ lives in the category of 
functors from finite pointed spaces to pointed spaces, it is the $n$-excisive approximation of the identity functor, and $\left(P_{n}(\text { id })\right)^{\text {inj }}$ denotes the fibrant replacement in the injective model structure constructed by Lurie in [33, Proposition A.3.3.2], see also Joyal [29], Jardine [28] for related work, and [10, Section 4] for the application to homotopy nilpotent groups. The simplicial set of morphisms $\mathcal{G}_{n}^{\text {op }}(k, l)$ is the space of natural transformations $\prod_{k} \Omega\left(P_{n}(\mathrm{id})\right)^{\mathrm{inj}} \rightarrow \prod_{l} \Omega\left(P_{n}(\mathrm{id})\right)^{\mathrm{inj}}$.

Hence, a pointed space $X$ is a homotopy nilpotent group of class $\leq n$ if it is the value at 1 of a simplicial functor $\underset{\sim}{X}$ from $\mathcal{G}_{n}$ to pointed spaces, which commutes up to homotopy with products. In fact, the homotopy nilpotent group is the whole functor $\underset{\sim}{X}$ and we isolate abusively the space $\underset{\sim}{X}(1)$, that comes in particular with a loop space structure since there is a structure map $\underset{\sim}{X}(1) \times \underset{\sim}{X}(1) \simeq \underset{\sim}{X}(2) \rightarrow \underset{\sim}{X}(1)$ corresponding to the loop space product. We say that $X$ is a homotopy nilpotent group if it is of class $\leq n$ for some $n$ and we write nil $l_{\mathrm{BD}}(X) \leq n$. The two extremes of this theory are well understood: loop spaces $\left(\operatorname{nil}_{\mathrm{BD}}(X) \leq \infty\right)$ and infinite loop spaces $\left(\operatorname{nil}_{\mathrm{BD}}(X) \leq 1\right.$, see $[10$, Theorem 5.13]) can be described as homotopy algebras over the theories $\mathcal{G}_{\infty}$ and $\mathcal{G}_{1}$ respectively.

\subsection{Nilpotency in the sense of Berstein-Ganea}

Applying the functor $\pi_{0}$ to the simplicial algebraic theory $\mathcal{G}_{n}$ gives us the ordinary theory of $n$-nilpotent groups, $\mathrm{Nil}_{n},[10$, Theorem 8.1]. Now, product preserving functors to the homotopy category of pointed spaces, $N: \mathrm{Nil}_{\mathrm{n}}^{\text {op }} \rightarrow \mathrm{Ho}\left(\operatorname{Spaces}_{*}\right)$, are, in other words, $\mathrm{Nil}_{n}$-algebras in the homotopy category of pointed spaces. These are exactly the $n$-nilpotent groups in the sense of Berstein-Ganea [14, Proposition 4.2]. Recall that for a loop space $\Omega Z$, the nilpotency of $\Omega Z$ in the sense of Berstein-Ganea is the least integer $n$ for which the $(n+1)$-st commutator map $\varphi_{n+1}:(\Omega Z)^{n+1} \rightarrow \Omega Z$ is homotopically trivial [8, Definition 1.7]; in this paper we write nil $l_{\mathrm{BG}}(\Omega Z) \leq n$. We recall here that if such an integer $n$ exists then all the $(n+1)$-fold iterated Whitehead products vanish in $Z$, [8, Theorem 4.6].

The following example shows that nilpotency in the sense of Berstein-Ganea does not capture the subtlety of the loop space structure.

Example 1.2. Let $f: \mathbb{C} P^{\infty} \rightarrow K(\mathbb{Z}, 6)$ represent the cube of the fundamental class of the infinite complex projective space, and let $Z$ be the homotopy fiber of $f$. The Berstein-Ganea nilpotency of $\Omega Z$ is 1 [37, Corollary 5.6] but its Biedermann-Dwyer nilpotency is strictly greater than 1 since $\Omega Z$ is not an infinite loop space ( $Z$ is not an $H$-space [37, Lemma 5.2]). Indeed, even though $\Omega Z$ is homotopically equivalent to a product of infinite loop spaces, $\Omega Z \simeq S^{1} \times K(\mathbb{Z}, 4)$, [37, Lemma 5.4], the homotopy does not preserve the loop structure. 
The next two invariants are recalled in the following paragraph since they are both related to fibrations, and in some sense, the second one is a refinement of the first.

\subsection{Inductive cocategory and epfl}

Different notions of cocategory exist; see, for example [26, Definition 2], [25, Definition p. 219], and [37, Definition 3.4]. In this paper, we concentrate on the inductive cocategory introduced by Ganea, [19, Definition 2.1], concretely, on its normalized version. Thus, for a connected pointed space $Z, \operatorname{cocat}(Z)=0$ if and only if $Z$ is contractible and, for any $n \geq 1, \operatorname{cocat}(Z) \leq n$ if there exists a fibration $F \rightarrow Y \rightarrow B$ with cocat $(Y) \leq n-1$ and $F$ dominates $Z$ (i.e. $Z$ is a retract of $F$ ); it is clear from the definition that $\operatorname{cocat}(F) \leq \operatorname{cocat}(Y)+1$. It is also clear that $\operatorname{cocat}(Z) \leq 1$ if and only if $Z$ is dominated by a loop space.

The relation between the cocategory and the nilpotency in the sense of Berstein-Ganea is given by the inequality $\operatorname{nil}_{\mathrm{BG}}(\Omega Z) \leq \operatorname{cocat}(Z),[19$, Theorem 2.12].

In this paper, we introduce a variant of the previous definition.

Definition 1.3. We say that a pointed connected space $Z$ is an extension by principal fibrations of length 0 if and only if $Z$ is contractible and, for $n \geq 1$, a space $Z$ is an extension by principal fibrations of length $\leq n$, if there exists a tower of principal fibrations

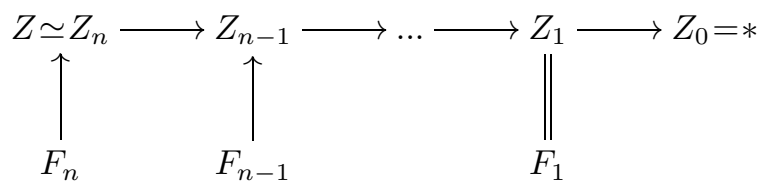

where $Z_{0}$ is a point and all fibers $F_{k}=\operatorname{Fib}\left(Z_{k} \rightarrow Z_{k-1}\right)$ are infinite loop spaces. In that case, we write $\operatorname{epfl}(Z) \leq n$.

Lemma 1.4. Let $Z$ be a pointed connected space. Then cocat $(Z) \leq \operatorname{epfl}(Z)$.

Proof. We prove the lemma by induction on $\operatorname{epfl}(Z)$. If $\operatorname{epfl}(Z)=1, Z$ is an infinite loop space, so $\operatorname{cocat}(Z) \leq 1$. If $\operatorname{epfl}(Z) \leq n$ for some integer $n>1$, by definition there exists a tower of principal fibrations (1) where the fibration $Z \rightarrow Z_{n-1}$ is classified by $\theta: Z_{n-1} \rightarrow B F_{n}$, that is, $Z$ is the homotopy fiber of $\theta$. Hence, $\operatorname{cocat}(Z) \leq$ $\operatorname{cocat}\left(Z_{n-1}\right)+1$ and, by induction, we get that $\operatorname{cocat}(Z) \leq \operatorname{cocat}(*)+n=n$.

We are now ready to prove the main theorem in this section. We exploit a characterization of homotopy nilpotent groups through excisive functors. For if $F$ 
is an $n$-excisive functor (so $F$ sends strongly homotopy co-Cartesian $(n+1)$-cubes to homotopy Cartesian ones, and also $F \simeq P_{n} F$ by [21, Theorem 1.8]) then, for every finite space $K, \Omega F(K)$ is a homotopy nilpotent group [10, Corollary 9.3]. Even better, Biedermann shows that every functor $\underset{\sim}{X}$ associated to a homotopy nilpotent group of class $\leq n$ is of the form $\Omega F$ where $F$ is $n$-excisive, [9].

We prove the following:

Theorem 1.5. Let $Z$ be a pointed connected space. Then, we have the inequalities

$$
\operatorname{nil}_{\mathrm{BG}}(\Omega Z) \leq \operatorname{cocat}(Z) \leq \operatorname{epfl}(Z) \leq \operatorname{nil}_{\mathrm{BD}}(\Omega Z) .
$$

Proof. In view of the previous paragraphs, nothing needs to be done for the first two inequalities. The first one is [19, Theorem 2.12] and the second one is Lemma 1.4. Suppose that $\Omega Z$ is a homotopy nilpotent group of class $\leq n$. Then, by [9], there exists an $n$-excisive functor $F$ and a space $K$ such that $\Omega Z$ and $\Omega F(K)$ are weakly equivalent as loop spaces. Therefore we have an equivalence $Z \simeq F(K)$. Now, as we mentioned in the Introduction, the Goodwillie tower for $F \simeq P_{n} F$ yields a tower

$$
F(K) \simeq P_{n} F(K) \longrightarrow P_{n-1} F(K) \longrightarrow \ldots \longrightarrow P_{1} F(K) \longrightarrow *
$$

whose fibers $D_{k} F(K)$ are infinite loop spaces, and $P_{k} F(K) \rightarrow P_{k-1} F(K)$ are principal fibrations classified by maps $P_{k-1} F(K) \rightarrow B D_{k} F(K)$, [21, Lemma 2.2]. This directly implies that $\operatorname{epfl}(Z) \leq n$.

When $\Omega Z$ is not only a loop space, but an infinite loop space, all inequalities are indeed equalities. This comes from the fact that homotopy 1-nilpotent groups are infinite loop spaces [10, Theorem 5.13].

Corollary 1.6. Let $X$ be an infinite loop space. Then $\operatorname{nil}_{\mathrm{BG}}(X)=\operatorname{nil}_{\mathrm{BD}}(X)=1$.

This approach leads us to an alternate and more direct proof of a result obtained in [14, Theorem 2.1] (see also Eldred's point of view, [18, Corollary 4.3]).

Corollary 1.7. Let $F$ be any n-excisive functor from the category of pointed spaces to pointed spaces. Then all $(n+1)$-fold iterated Whitehead products vanish in $F(K)$ for every finite space $K$.

Proof. Since $F$ is an $n$-excisive functor, $\Omega F(K)$ is a homotopy nilpotent group of class $\leq n,[10$, Theorem 9.2]. Thus nil $\mathrm{BG}(\Omega F(K)) \leq n$ by Theorem 1.5 and all the $(n+1)$-fold iterated Whitehead products vanish in $F(K)$ (see Section 1.2).

This application highlights the simplicity of the arguments when using the relationship between the classical notions of nilpotency, nil ${ }_{\mathrm{BG}}$ and cocat, and the more recent ones, epfl and nil $\mathrm{BD}$. 
Remark 1.8. The upper bound we obtain in Corollary 1.7 is a crude estimate. In [4, Theorem 4.2] Arone, Dwyer, and Lesh show that any (2n-1)-excisive spacevalued functor $F$, for which $P_{n-1} F \simeq *$, takes its values in infinite loop spaces. Therefore, since nil $\mathrm{BG}_{\mathrm{BG}} F(K) \leq 1$, all Whitehead products vanish in $F(K)$, whereas by Corollary 1.7 we would only obtain that the $2 n$-fold iterated Whitehead products vanish.

We end this section by showing that all nilpotency notions coincide for discrete groups. In order to do so, we use the relation of the simplicial algebraic theory $\mathcal{G}_{n}$ with the set-valued theory of ordinary $n$-nilpotent groups $\mathrm{Nil}_{n}$.

Theorem 1.9. Let $G$ be a discrete nilpotent group. Then

$$
\operatorname{nil}_{\mathrm{BG}}(G)=\operatorname{cocat}(B G)=\operatorname{epfl}(B G)=\operatorname{nil}_{\mathrm{BD}}(G) .
$$

Proof. Let $G$ be a nilpotent group of class $n$, i.e. $n=\operatorname{nil}_{\mathrm{BG}}(G)$. There exists then a set valued $\mathrm{Nil}_{n}$-algebra given by the functor $G: \mathrm{Nil}_{n} \rightarrow \mathrm{Sets}_{*}$ where $G(k)=G^{k}$. Considering the isomorphism of categories $\mathrm{Nil}_{n} \cong \pi_{0} \mathcal{G}_{n}[10$, Theorem 8.1] and viewing pointed sets as a full subcategory of $\mathcal{S}_{*}$, shows that $G$ is a homotopy

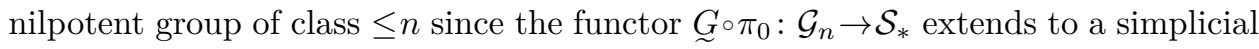
functor as all mapping spaces between discrete groups are discrete. It is therefore a homotopy $\mathcal{G}_{n}$-algebra and

$$
n=\operatorname{nil}_{\mathrm{BG}}(G) \leq \operatorname{cocat}(B G) \leq \operatorname{epfl}(B G) \leq \operatorname{nil}_{\mathrm{BD}}(G) \leq n .
$$

Remark 1.10. We would be remiss in not saying a word about examples of spaces for which these inequalities are sharp. All of our attempts have been unsuccessful to find an example of a space $X$ for which epfl $(X)$ is strictly less than $\operatorname{nil}_{\mathrm{BD}}(\Omega X)$.

\section{Towers of principal fibrations and Neisendorfer's type functor}

The aim of this section is to show that the effect of certain homotopical localization functors on loop spaces with finite epfl, see Definition 1.3, is predictable. We start with a short subsection where we fix the notation and terminology about localization and cellularization. Most of this is taken from the first chapters in [15]. It is convenient to work here in the category of simplicial sets (which we call spaces). From now on $p$ denotes a prime number.

\subsection{Localization and cellularization}

Let $f: A \rightarrow B$ be a map. A space $X$ is $f$-local if $\operatorname{map}(f, X)$ is a weak equivalence. A map $g$ is an $f$-local equivalence if $\operatorname{map}(g, X)$ is a weak equivalence for all $f$-local 
spaces $X$. There exists a coaugmented homotopy idempotent functor $\mathrm{L}_{f}$ called $f$-localization, [15, Theorem 1.A.3], such that the coaugmentation map $\eta: X \rightarrow \mathrm{L}_{f} X$ is an $f$-local equivalence to an $f$-local space.

Example 2.1. When $f$ is a map of the form $A \rightarrow *$, one traditionally writes $\mathrm{P}_{A}$ for the localization functor $\mathrm{L}_{f}$. This functor is called $A$-nullification. It turns $A$ into a point and one says that $\mathrm{P}_{A}$ kills $A$. For $A=S^{n+1}$ one obtains a functorial construction of the $n$-th Postnikov section since $\mathrm{P}_{S^{n+1}} X \simeq X[n]$, [15, Example 1.A.1.1]. We will also be interested in $B \mathbb{Z} / p$-nullification.

Example 2.2. Let $f$ be a universal $H \mathbb{F}_{p}$-equivalence, meaning that $f$ is a wedge of all maps between countable simplicial sets that induce an isomorphism in mod $p$ homology. Then $\mathrm{L}_{f}$ is $\bmod p$ homological localization, [15, Example 1.E.4]. The effect of $\mathrm{L}_{f}$ on nilpotent spaces is, up to homotopy, Bousfield-Kan $p$-completion, [11].

We turn now to the description of the localization functor we need. We combine $B \mathbb{Z} / p$-nullification and $H \mathbb{F}_{p}$-localization so as to obtain a functor that "kills" $B \mathbb{Z} / p$ and "inverts" all mod $p$ homology equivalences.

Definition 2.3. Let $f: B \mathbb{Z} / p \rightarrow *$ and $g$ be a universal $H \mathbb{F}_{p}$-equivalence. We set $\mathrm{L}=\mathrm{L}_{f \vee g}$.

The reason behind this definition is that $\mathrm{L}$ is a homotopy idempotent version of Neisendorfer's functor $\left(\mathrm{P}_{B \mathbb{Z} / p}(-)\right)_{p}^{\wedge},[38$, Section 1], BZ/Z/p-nullification followed by $p$-completion. Note that, by the universal properties of the functors, we have a natural transformation of coaugmented functors $\left(\mathrm{P}_{B \mathbb{Z} / p}(-)\right)_{p}^{\wedge} \rightarrow \mathrm{L}$. This induces a weak equivalence $\left(\mathrm{P}_{B \mathbb{Z} / p} X\right)_{p}^{\wedge} \stackrel{\sim}{\longrightarrow} \mathrm{L} X$ whenever $\mathrm{P}_{B \mathbb{Z} / p} X$ is a nilpotent space since in that case we know that $\left(\mathrm{P}_{B \mathbb{Z} / p} X\right)_{p}^{\wedge}$ is $B \mathbb{Z} / p$-null.

Remark 2.4. If $X$ is a connected infinite loop space with a torsion fundamental group, then $\mathrm{L} X$ is contractible. Indeed, McGibbon proved that for such spaces, $\left(\mathrm{P}_{B \mathbb{Z} / p} X\right)_{p}^{\wedge}$ is contractible, [35, Theorem 2]. As infinite loop spaces are nilpotent, we have also that $\mathrm{P}_{B \mathbb{Z} / p} X$ is nilpotent and therefore, by the comments above $\mathrm{L} X \simeq$ $\left(\mathrm{P}_{B \mathbb{Z} / p} X\right)_{p}^{\wedge} \simeq *$. In general, however, $\mathrm{L}$ and $\left(\mathrm{P}_{B \mathbb{Z} / p}(-)\right)_{p}^{\wedge}$ differ.

The functorial nature of homotopy localization is quite powerful, as is illustrated by the following central property.

Theorem 2.5. ([15, Theorem 1.H.1]) If $F \rightarrow E \stackrel{p}{\longrightarrow} B$ is a fibration and $\mathrm{L}_{f} F \simeq *$, then $\mathrm{L}_{f}(p): \mathrm{L}_{f} E \rightarrow \mathrm{L}_{f} B$ is a homotopy equivalence. 
We end this subsection by introducing cellularization functors. We fix a pointed space $A$. A pointed space $X$ is $A$-cellular if it belongs to the smallest class of pointed spaces containing $A$ and closed under weak equivalences and pointed homotopy colimits, [15, Definition 2.D.1]. A pointed map $f$ is an $A$-equivalence if $\operatorname{map}_{*}(A, f)$ is a weak equivalence. There exists an augmented homotopy idempotent functor $\mathrm{CW}_{A}$ called $A$-cellularization such that the augmentation map $\varepsilon: \mathrm{CW}_{A} X \rightarrow X$ is an $A$-equivalence from an $A$-cellular space, [15, Theorem 2.B.3].

Example 2.6. When $A=S^{n+1}$, then $\mathrm{CW}_{S^{n+1}} X$ is a functorial analogue of $X\langle n\rangle$, the $n$-connected cover of $X,\left[15\right.$, Example 2.D.2.6]. Thus $\mathrm{CW}_{S^{n+1}} X$ coincides with the homotopy fiber of the coaugmentation $X \rightarrow \mathrm{P}_{S^{n+1}} X$. In particular, when $n=1$, we get a functorial construction of the universal cover.

Farjoun shows in [15, Theorems 3.A.1 and 3.A.2] that localization and cellularization functor behave well with respect to loop space structures. His statements and the proofs are more precise than the following theorem, which will be sufficient for us.

Theorem 2.7. (Farjoun, [15]) For any map $f$ and any pointed space $A$, the coaugmentation $\Omega X \rightarrow \mathrm{L}_{f} \Omega X$ and the augmentation $\mathrm{CW}_{A} \Omega X \rightarrow \Omega X$ are homotopic to loop maps, i.e. maps between loop spaces preserving the loop space structure.

\subsection{The effect of $L$ on certain loop spaces}

We work with the functor $\mathrm{L}$ introduced in Definition 2.3. We first show that the effect of $\mathrm{L}$ on loop spaces can be seen in the universal cover and second, that the epfl of the universal cover is not greater than that of the space.

Lemma 2.8. Let $X$ be a connected space such that $\pi_{1} X$ is a finite group, and let $X\langle 1\rangle$ be the universal cover of $X$. If $\mathrm{L}(X\langle 1\rangle)$ is contractible, then so is $\mathrm{L} X$.

Proof. Consider the fibration sequence $X\langle 1\rangle \rightarrow X \rightarrow B \pi_{1} X$ given by the universal cover. As we assume that $\mathrm{L}(X\langle 1\rangle)$ is contractible, Theorem 2.5 implies that $\mathrm{L} X \simeq \mathrm{L}\left(B \pi_{1} X\right)$. But $\mathrm{P}_{B \mathbb{Z} / p}\left(B \pi_{1} X\right)$ is contractible by [22, Lemma 6.6], hence so are $\mathrm{L}\left(B \pi_{1} X\right)$ and $\mathrm{L} X$.

Lemma 2.9. Let $f: Z \rightarrow Y$ be a map of connected spaces whose homotopy fiber $F$ is an infinite loop space. Then the homotopy fiber of the induced map on universal covers $f\langle 1\rangle: Z\langle 1\rangle \rightarrow Y\langle 1\rangle$ is an infinite loop space as well. Moreover, if $f$ is a principal fibration, then so is $f\langle 1\rangle$. 
Proof. Consider the horizontal ladder of fibration sequences

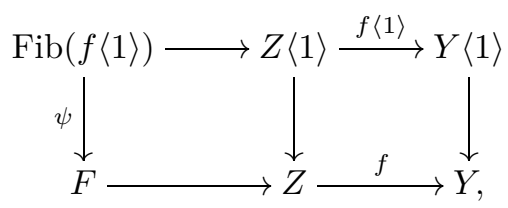

and the associated ladder of long exact homotopy sequences. By the Five Lemma, we obtain that $\pi_{r}(\psi)$ is an isomorphism for $r \geq 2$, while the Snake Lemma shows that $\pi_{1}(\psi)$ is a monomorphism. Moreover, since $\operatorname{Fib}(f\langle 1\rangle)$ is connected, the range of $\psi$ is in $F_{c}$, the base point component of $F$, and the long exact homotopy sequence of the fibration

$$
\operatorname{Fib} \psi \longrightarrow \operatorname{Fib}(f\langle 1\rangle) \stackrel{\psi}{\longrightarrow} F_{c}
$$

shows that Fib $\psi$ is a homotopically discrete space. Then, $\operatorname{Fib}(f\langle 1\rangle)$ is a covering of $F_{c}$, and therefore it is an infinite loop space since infinite loop structures are preserved when considering connected components and covers (see Theorem 2.7). Moreover, if $f$ is a principal fibration, so is $f\langle 1\rangle$, since taking universal covers is a particular case of cellularization (see Example 2.6) and, the cellularization of a principal fibration is again a principal fibration by [16, Theorem 2.1].

Corollary 2.10. Let $Z$ be a pointed connected space. Then, epfl $(Z\langle 1\rangle) \leq$ $\operatorname{epfl}(Z)$.

Proof. Suppose that $Z$ is an extension by principal fibrations of length $n$ and apply, to the associated tower of principal fibrations, the universal cover functor. Then, the result follows by Lemma 2.9.

We refine this elementary observation to obtain a version where the homotopy fibers in the tower are simply connected, a key technical fact for what follows.

Lemma 2.11. Let $f: Z \rightarrow Y$ be a principal fibration of simply connected spaces whose homotopy fiber $F$ is an infinite loop space. There exists then a factorization $f: Z \rightarrow \bar{Y} \rightarrow Y$ such that the homotopy fiber $\operatorname{Fib}(Z \rightarrow \bar{Y})$ is $F\langle 1\rangle$, the universal cover of $F$.

Proof. Let $\theta: Y \rightarrow B F$ denote the classifying map of the principal fibration $f$. Since $F$ is connected, $B F$ is simply connected and the second stage of the Postnikov tower becomes

$$
B(F\langle 1\rangle) \simeq(B F)\langle 2\rangle \longrightarrow B F \stackrel{\varkappa_{2}}{\longrightarrow} K\left(\pi_{2} B F, 2\right) .
$$

Let $\bar{Y}$ be the homotopy fiber of $\varkappa_{2} \circ \theta: Y \rightarrow K\left(\pi_{2} B F, 2\right)$. Since $\varkappa_{2} \circ \theta \circ f \simeq *$, then $f$ factors through $\bar{f}: Z \rightarrow \bar{Y}$. Loop now the existing fibration sequences to obtain the 
following commutative diagram of horizontal and vertical fibration sequences:

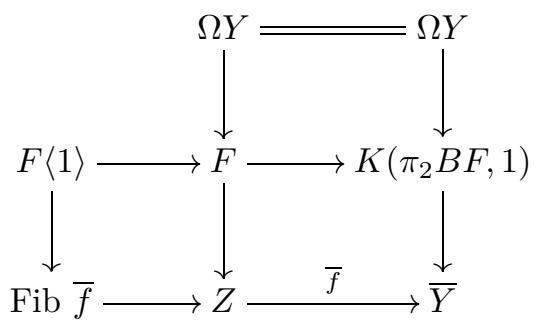

Since the lower right square is a homotopy pull-back (principal fibrations with the same vertical fiber), the map between the horizontal fibers $F\langle 1\rangle \rightarrow$ Fib $\bar{f}$ is a homotopy equivalence.

The following result is the final step that will allow us to understand the effect of the functor $\mathrm{L}$ on a space $Z$ with finite epfl.

Proposition 2.12. Let $Z$ be a connected space with $\operatorname{epfl}(Z) \leq n$. Then, $Z\langle 1\rangle$ is also an extension by (not necessarily principal) fibrations of length $n$, where the fibers are simply connected infinite loop spaces.

Proof. Since $\operatorname{epfl}(Z) \leq n$ there exists a tower of principal fibrations

$$
Z \simeq Z_{n} \longrightarrow Z_{n-1} \longrightarrow \ldots \longrightarrow Z_{1} \longrightarrow *
$$

such that the homotopy fibers $\operatorname{Fib}\left(Z_{k} \rightarrow Z_{k-1}\right)$ are infinite loop spaces. Since $Z$ is connected the spaces $Z_{k}$ can be chosen to be connected as well (notice that fibrations are, in particular, surjective maps). Lemma 2.9 implies that the tower of universal covers is made of principal fibrations that have also infinite loop spaces as homotopy fibers. We are thus left with a tower of simply connected spaces

$$
Z\langle 1\rangle \simeq Z_{n}\langle 1\rangle \longrightarrow Z_{n-1}\langle 1\rangle \longrightarrow \ldots \longrightarrow Z_{1}\langle 1\rangle \longrightarrow *
$$

The fibers $F_{k}$ however are not simply connected in general, but only connected. We modify thus the spaces $Z_{k}\langle 1\rangle$ for $k<n$ to find a different and more convenient tower for $Z\langle 1\rangle$. Since $F_{1}=Z_{1}\langle 1\rangle$ is simply connected, we can assume by induction that $Z_{n-1}\langle 1\rangle$ is an extension by fibrations of length $n-1$, with simply connected infinite loop spaces as fibers.

We use now the factorization $Z_{n}\langle 1\rangle \rightarrow \overline{Z_{n-1}\langle 1\rangle} \rightarrow Z_{n-1}\langle 1\rangle$ of Lemma 2.11. Since the homotopy fiber of the first map is a simply connected infinite loop space, to conclude the proof we must show that the space in the middle is an extension by fibrations of length $n-1$ with simply connected infinite loop spaces as fibers. Consider now the homotopy fiber $H$ of the composite map $\overline{Z_{n-1}\langle 1\rangle} \rightarrow Z_{n-1}\langle 1\rangle \rightarrow$ 
$Z_{n-2}\langle 1\rangle$. The homotopy fiber $H$ fits by construction into a fibration sequence

$$
H \longrightarrow F_{n-1} \longrightarrow K\left(\pi_{2} B F_{n}, 2\right) \text {. }
$$

However, since $F_{n-1}$ is simply connected by induction hypothesis, the map $F_{n-1} \rightarrow$ $K\left(\pi_{2} B F_{n}, 2\right)$ factors through the second Postnikov section of $F_{n-1}$. This is a map of infinite loop spaces, see Theorem 2.7, which implies that $H$ is an infinite loop space. Therefore, the tower $\overline{Z_{n-1}\langle 1\rangle} \rightarrow Z_{n-2}\langle 1\rangle \rightarrow \ldots \rightarrow Z_{1}\langle 1\rangle$ exhibits $\overline{Z_{n-1}\langle 1\rangle}$ as an extension by fibrations of length $n-1$ with connected infinite loop spaces as fibers. The induction hypothesis allows us to conclude that it is also an extension by principal fibrations of length $n-1$ with simply connected infinite loop spaces as fibers.

We finally describe the effect of L (see Definition 2.3) on loop spaces of finite extension by principal fibrations length:

Theorem 2.13. Let $\Omega Z$ be a connected space with finite fundamental group. If $\operatorname{epfl}(Z)$ is finite, then $\mathrm{L}(\Omega Z)$ is contractible.

Proof. By Lemma 2.8 it is enough to prove that $\mathrm{L}((\Omega Z)\langle 1\rangle)$ is contractible. Let $\operatorname{epfl}(Z) \leq n$. Then, since looping a tower of fibrations with infinite loop spaces fibers yields another such tower, $\operatorname{epf}(\Omega Z) \leq n$ and by Proposition $2.12,(\Omega Z)\langle 1\rangle$ is also an extension by fibrations of length $n$,

$$
(\Omega Z)\langle 1\rangle \simeq Y_{n} \longrightarrow Y_{n-1} \longrightarrow \ldots \longrightarrow Y_{1} \longrightarrow *
$$

where the fibers are simply connected infinite loop spaces $F_{k}$. By Remark 2.4, L $F_{k}$ is contractible for any $n \geq k \geq 1$, and according to Theorem $2.5, \mathrm{~L}\left(Y_{k}\right) \simeq \mathrm{L}\left(Y_{k-1}\right)$. Therefore, an inductive argument shows that $\mathrm{L}((\Omega Z)\langle 1\rangle) \simeq *$.

Remark 2.14. The finiteness assumption on the fundamental group in Theorem 2.13 is not necessary, but the contractibility of $\mathrm{L}(\Omega Z)$ does not hold as soon as there is a copy of the integers in $\pi_{1}(\Omega Z)$. Suppose for example that $\pi_{1}(\Omega Z)$ is a finitely generated abelian group which is infinite. There exists then an epimorphism to $\mathbb{Z}$ which can be used to construct a map $\Omega Z \rightarrow K\left(\pi_{1}(\Omega Z), 1\right) \rightarrow K(\mathbb{Z}, 1)=S^{1}$. This map has a section hence $\Omega Z$ dominates $S^{1}$. Therefore $\mathrm{L}(\Omega Z)$ dominates $\mathrm{L} S^{1} \simeq\left(S^{1}\right)_{p}^{\wedge}$, and $\mathrm{L}(\Omega Z)$ is not contractible.

\section{Homotopy nilpotency of $p$-complete loop spaces}

In this section we characterize homotopy commutative loop spaces in the sense of Biedermann-Dwyer, and more generally homotopy nilpotent groups with finiteness conditions. 
We first recall some definitions. A loop space $(X, B X)$ is said to be a $p$-compact group [17, Definition 2.3] if $B X$ is $p$-complete and $X$ is $\mathbb{F}_{p}$-finite, i.e., $H^{*}\left(X ; \mathbb{F}_{p}\right)$ is

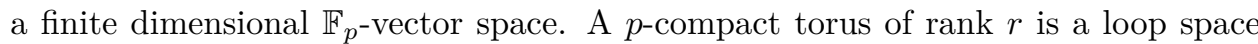
$(T, B T)$ such that $B T$ is an Eilenberg-MacLane space of type $K\left(\left(\mathbb{Z}_{p}^{\wedge}\right)^{r}, 2\right)[17$, Definition 6.3]. The following result characterizes homotopy nilpotent $p$-compact groups in the Biedermann-Dwyer sense.

Theorem 3.1. Let $(X, B X)$ be a connected $p$-compact group. Then, $\operatorname{nil}_{\mathrm{BD}}(X)$ is finite if and only if $(X, B X)$ is a p-compact torus.

Proof. By applying the cellularization functor $\mathrm{CW}_{S^{2}}$ to $X$, we obtain the universal cover of $X, \mathrm{CW}_{S^{2}}(X)=X\langle 1\rangle \simeq \mathrm{Fib}\left(X \rightarrow B \pi_{1}(X)\right)$. Cellularization is a continuous functor that preserves products up to homotopy [15, Theorem 2.E.10], therefore if $\operatorname{nil}_{\mathrm{BD}}(X)$ is finite, then so is $\operatorname{nil}_{\mathrm{BD}}(X\langle 1\rangle)$. Now, since $X\langle 1\rangle$ is a connected loop space with finite fundamental group, Theorem 2.13 tells us that $\mathrm{L}(X\langle 1\rangle)$ must be contractible.

However, by Miller's proof of the Sullivan conjecture [36, Theorem A] the pointed mapping space $\operatorname{map}_{*}(B \mathbb{Z} / p, X\langle 1\rangle)$ is contractible. In terms of localization functors, this means that $X\langle 1\rangle$ is $B \mathbb{Z} / p$-local, and so $\mathrm{P}_{B \mathbb{Z} / p}(X\langle 1\rangle) \simeq X\langle 1\rangle$, as noticed already in the introduction of [38]. Therefore, since $X\langle 1\rangle$ is $p$-complete, it is in fact $(f \vee g)$-local, which means that $\mathrm{L}(X\langle 1\rangle) \simeq X\langle 1\rangle$. Thus, $X\langle 1\rangle$ must be contractible, so that $X$ is homotopy equivalent to $B \pi_{1}(X)$.

Finally, by [17, Remark 2.2], the fundamental group of a $p$-compact group is a finite direct sum of copies of cyclic groups $\mathbb{Z} / p^{r}$ and copies of $p$-adic integers $\mathbb{Z}_{p}^{\wedge}$. Since $X$ is $\mathbb{F}_{p}$-finite, and $B \mathbb{Z} / p^{r}$ is not, this implies that $\pi_{1}(X)$ contains no factor of $\mathbb{Z} / p^{r}$-type. We conclude that $(X, B X)$ is a $p$-compact torus.

For any simply connected Lie group $G$, the $p$-completion of $B G$ gives rise to a $p$-compact group. The following result is thus straightforward.

Corollary 3.2. Let $G$ be a non-trivial simply connected compact Lie group. Then $\operatorname{nil}_{\mathrm{BD}}\left(G_{p}^{\wedge}\right)$ is infinite.

This implies that the nilpotency in the sense of Berstein-Ganea, and the nilpotency in the sense of Biedermann-Dwyer do not coincide in general.

Example 3.3. McGibbon's p-local examples of classically homotopy commutative compact Lie groups can be translated into the $p$-complete setting, [34, Theorem 2]. Simply connected simple Lie groups at large enough primes, $S p(2)$ at the prime 3, and the exceptional group $G_{2}$ at the prime 5, have Berstein-Ganea nilpotency 1 , whereas according to Corollary 3.2 they all have infinite BiedermannDwyer nilpotency. 
In [30], Kaji and Kishimoto study examples of $p$-compact groups that are nilpotent in the sense of Berstein-Ganea but they are not $p$-compact tori, so by Theorem 3.1 they are not homotopy nilpotent in the sense of Biedermann-Dwyer. There exist, in fact, infinitely many loop spaces $X$ for which nil ${ }_{\mathrm{BG}}(X)<\infty$, but $\operatorname{nil}_{\mathrm{BD}}(X)=\infty$. This is the case for $E_{8}$ at the prime 41 with nil ${ }_{\mathrm{BG}}\left(\left(E_{8}\right)_{41}^{\wedge}\right)=3$, [30, Theorem 1.6].

We move now from $p$-compact groups to $p$-Noetherian groups. Let us recall from [13] that a $p$-Noetherian group is a loop space $(X, B X)$ where $B X$ is $p$-complete and $H^{*}\left(X ; \mathbb{F}_{p}\right)$ is a finitely-generated (Noetherian) $\mathbb{F}_{p}$-algebra.

Theorem 3.4. Let $(X, B X)$ be a connected $p$-Noetherian group. If $X$ is a homotopy nilpotent group, then $B X$ fits in a fibration sequence

$$
K(Q, 2) \times K\left(\mathbb{Z}_{p}^{\wedge}, 3\right)^{r} \longrightarrow B X \longrightarrow\left(\left(B S^{1}\right)_{p}^{\wedge}\right)^{s}
$$

where $Q$ is a finite abelian p-group, and $r, s \geq 0$.

Proof. In [13, Theorem 1.9], for a $p$-Noetherian group $X$, the authors construct a fibration

$$
K(P, 2)_{p}^{\wedge} \longrightarrow B X \longrightarrow B Y=\left(\mathrm{P}_{\Sigma B \mathbb{Z} / p} B X\right)_{p}^{\wedge}
$$

where $P=Q \oplus\left(\mathbb{Z} / p^{\infty}\right)^{r}$, for $Q$ a finite abelian $p$-group, $r \geq 0, \mathbb{Z} / p^{\infty}=\mathbb{Z}[1 / p] / \mathbb{Z}$ is a Prüfer group, [42, Theorem 10.13], and $(Y, B Y)$ is a $p$-compact group. Since we have a series of homotopy equivalences

$$
Y \simeq \Omega B Y \simeq \Omega\left(\mathrm{P}_{\Sigma B \mathbb{Z} / p} B X\right)_{p}^{\wedge} \simeq\left(\Omega\left(\mathrm{P}_{\Sigma B \mathbb{Z} / p} B X\right)\right)_{p}^{\wedge}
$$

and $\left(\Omega\left(\mathrm{P}_{\Sigma B \mathbb{Z} / p} B X\right)\right)_{p}^{\wedge}$ is homotopy equivalent to $\left(\mathrm{P}_{B \mathbb{Z} / p} \Omega B X\right)_{p}^{\wedge}$ by $[15$, Theorem 3.A.1], we get that $Y \simeq\left(\mathrm{P}_{B \mathbb{Z} / p} X\right)_{p}^{\wedge}$. Also observe that all the homotopy equivalences are loop maps by Theorem 2.7. This allows us to say that $(Y, B Y)$ is obtained in a functorial way from $(X, B X)$ using the nullification and the $p$-completion functors. Both functors are continuous and preserve products up to homotopy. Therefore, if $\operatorname{nil}_{\mathrm{BD}}(X)$ is finite, so is $\operatorname{nil}_{\mathrm{BD}}(Y)$.

Finally, since $(Y, B Y)$ is a $p$-compact group and $\operatorname{nil}_{\mathrm{BD}}(Y)$ is finite, Theorem 3.1 implies that $(Y, B Y)$ must be a $p$-compact torus, that is, $B Y \simeq\left(\left(B S^{1}\right)_{p}^{\wedge}\right)^{s}$ for some $s \geq 0$. For $\mathbb{Z} / p^{\infty}, p$-completion shifts dimension by one, [11, Example VI.6.4]:

$$
K(P, 2)_{p}^{\wedge} \simeq K(Q, 2) \times K\left(\left(\mathbb{Z} / p^{\infty}\right)^{r}, 2\right)_{p}^{\wedge} \simeq K(Q, 2) \times K\left(\mathbb{Z}_{p}^{\wedge}, 3\right)^{r}
$$

and the result follows.

It follows from the previous theorem that $p$-Noetherian groups, which are homotopy nilpotent, have epfl at most 2, where the tower of principal fibrations with 
infinite loop space fibers is provided by the Postnikov tower. We prove now that this invariant coincides with the Biedermann-Dwyer nilpotency in this situation.

Corollary 3.5. Let $(X, B X)$ be a connected $p$-Noetherian group and assume that $\operatorname{nil}_{\mathrm{BD}}(X)$ is finite. Then $\operatorname{nil}_{\mathrm{BD}}(X) \leq 2$.

Proof. By the previous theorem, the homotopy groups $\pi_{n}(B X)$ vanish unless $2 \leq n \leq 3$. The non-trivial homotopy groups of the simply connected space $B X$ live thus in the metastable range, therefore by [10, Example 9.8], nil $\mathrm{BD}(X) \leq 2$.

We finally come back to the integral Torus Theorem, [27, Theorem 1.1]. The classical statement is that, if $\operatorname{nil}_{\mathrm{BG}}(X)=1$, then $X$ has the homotopy type of a torus.

Theorem 3.6. Let $(X, B X)$ be a connected finite loop space. If $\operatorname{nil}_{\mathrm{BD}}(X)$ is finite, then $X$ has the homotopy type of a torus.

Proof. For a finite and connected loop space $(X, B X)$, it is well known that the $p$-completion $\left(X_{p}^{\wedge}, B X_{p}^{\wedge}\right)$ is a $p$-compact group for every prime $p$ (see, for example, $\left[3\right.$, Introduction] and [23, p. 990]). Theorem 3.1 applies and we obtain that $X_{p}^{\wedge}$ is a $p$-compact torus for every prime $p$. Now, Sullivan's arithmetic square, [11, Theorem VI.8.1],

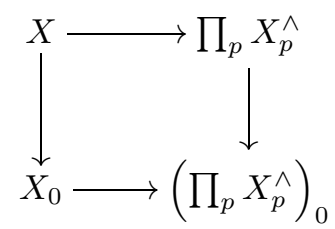

is a homotopy pull-back square. On the right hand side, we have a product of $p$-complete tori and its rationalization, whereas on the bottom left corner the rationalization of a finite loop space is a product of odd dimensional rational spheres $K(\mathbb{Q}, 2 k+1)$. Any higher dimensional sphere than $S^{1}$ would remain in the homotopy pull-back, so $X_{0}$ must be a rational torus and we conclude that $X$ has the homotopy type of a torus.

Following the ideas of Rector [41], it is commonly accepted that compact Lie groups should be thought of as finite loop spaces, and that the structural data of the Lie group have to be read by means of homotopy invariants. Within this framework, connected non-abelian compact Lie groups are expected to be highly non-nilpotent. The previous result shows that nilpotency in the sense of Biedermann-Dwyer is the right notion in contrast with classical nilpotency in the sense of Berstein-Ganea. So for example, by a classical result of Porter [39] the sphere $S^{3}$ is nilpotent in the sense of Berstein-Ganea, $\operatorname{nil}_{\mathrm{BG}}\left(S^{3}\right)=3$, but since $S^{3}$ is not a torus, $\operatorname{nil}_{\mathrm{BD}}\left(S^{3}\right)=\infty$. 


\section{References}

1. Adem, A. and Milgram, R. J., Cohomology of Finite Groups, 2nd ed., Grundlehren der Mathematischen Wissenschaften 309, Springer, Berlin, 2004, viii+324 pp.

2. Aguadé, J. and Smith, L., On the mod $p$ torus theorem of John Hubbuck, Math. Z. 191 (1986), 325-326.

3. Andersen, K. K. S., Bauer, T., Grodal, J. and Pedersen, E. P., A finite loop space not rationally equivalent to a compact Lie group, Invent. Math. 157 (2004), 1-10.

4. Arone, G., Dwyer, W. G. and Lesh, K., Loop structures in Taylor towers, Algebr. Geom. Topol. 8 (2008), 173-210.

5. Arone, G. and Mahowald, M., The Goodwillie tower of the identity functor and the unstable periodic homotopy of spheres, Invent. Math. 135 (1999), $743-788$.

6. Badzioch, B., Algebraic theories in homotopy theory, Ann. of Math. 155 (2002), 895-913.

7. Berrick, A. J., An Approach to Algebraic K-Theory, Research Notes in Mathematics 56, Pitman (Advanced Publishing Program), Boston, Mass.-London, 1982, iii +108 pp.

8. Berstein, I. and Ganea, T., Homotopical nilpotency, Illinois J. Math. 5 (1961), 99-130.

9. Biedermann, G., Homotopy nilpotent groups and their associated functors, Preprint. arXiv. org/abs/1705.04963.

10. Biedermann, G. and Dwyer, W. G., Homotopy nilpotent groups, Algebr. Geom. Topol. 10 (2010), 33-61.

11. Bousfield, A. K. and KAn, D. M., Homotopy Limits, Completions and Localizations, Lecture Notes in Mathematics 304, Springer, Berlin-New York, 1972, $\mathrm{v}+348 \mathrm{pp}$.

12. Castellana, N., Crespo, J. and Scherer, J., Deconstructing Hopf spaces, Invent. Math. 167 (2007), 1-18.

13. Castellana, N., Crespo, J. and Scherer, J., Noetherian loop spaces, J. Eur. Math. Soc. 13 (2011), 1225-1244.

14. Chorny, B. and Scherer, J., Goodwillie calculus and Whitehead products, Forum Math. 27 (2015), 119-130.

15. Dror Farjoun, E., Cellular Spaces, Null Spaces and Homotopy Localization, Lecture Notes in Mathematics 1622, Springer, Berlin, 1996.

16. Dwyer, W. G. and FarJoun, E. D., Localization and cellularization of principal fibrations, in Alpine Perspectives on Algebraic Topology, Contemp. Math. 504, pp. 117-124, Amer. Math. Soc., Providence, RI, 2009.

17. Dwyer, W. and Wilkerson, C., Homotopy fixed points methods for Lie groups and finite loop spaces, Ann. of Math 139 (1994), 395-442.

18. Eldred, R., Goodwillie calculus via adjunction and LS cocategory, Homology, Homotopy Appl. 18 (2016), 31-58.

19. Ganea, T., Lusternik-Schnirelmann category and cocategory, Proc. Lond. Math. Soc. 10 (1960), 623-639.

20. Goodwillie, T. G., Calculus. II. Analytic functors, K-Theory 5 (1991/92), 295-332. 
21. Goodwillie, T. G., Calculus. III. Taylor series, Geom. Topol. 7 (2003), 645-711 (electronic).

22. Grodal, J., The transcendence degree of the mod p cohomology of finite Postnikov systems, in Stable and Unstable Homotopy, Fields Inst. Commun. 19, pp. 111$130,1998$.

23. Grodal, J., The classification of $p$-compact groups and homotopical group theory, in Proceedings of the International Congress of Mathematicians. Volume II, pp. 973-1001, Hindustan Book Agency, New Delhi, 2010.

24. Hopkins, M. J., Formulations of cocategory and the iterated suspension, in Algebraic Homotopy and Local Algebra, Astérisque 113-114, pp. 212-226, 1984.

25. Hopkins, M. J., Nilpotence and finite $H$-spaces, Israel J. Math. 66 (1989), 238-246.

26. Hovey, M., Lusternik-Schnirelmann cocategory, Illinois J. Math. 37 (1993), 224-239.

27. Hubbuck, J. R., On homotopy commutative $H$-spaces, Topology 8 (1969), 119-126.

28. Jardine, J. F., Simplicial presheaves, J. Pure Appl. Algebra 47 (1987), 35-87.

29. Joyal, A., Letter to A. Grothendieck. https://webusers.imj-prg.fr/ georges. maltsiniotis/ps/lettreJoyal.pdf.

30. Kaji, S. and Kishimoto, D., Homotopy nilpotency in p-regular loop spaces, Math. Z. 264 (2010), 209-224.

31. Lawvere, F. W., Functorial semantics of algebraic theories, Proc. Natl. Acad. Sci. USA 50 (1963), 869-872.

32. Lin, J., A cohomological proof of the torus theorem, Math. Z. 190 (1985), 469-476.

33. Lurie, J., Higher Topos Theory, Annals of Mathematics Studies 170, Princeton University Press, Princeton, NJ, 2009, xviii+925 pp.

34. McGibbon, C. A., Homotopy commutativity in localized groups, Amer. J. Math. 106 (1984), 665-687.

35. McGibbon, C. A., Infinite loop spaces and Neisendorfer localization, Proc. Amer. Math. Soc. 125 (1997), 309-313.

36. Miller, H., The Sullivan conjecture on maps from classifying spaces, Ann. of Math. 120 (1984), 39-87.

37. Murillo, A. and Viruel, A., Lusternik-Schnirelmann cocategory: A Whitehead dual approach, in Cohomological Methods in Homotopy Theory, Progr. Math. 196, pp. 323-347, Birkhäuser Verlag, Basel, 2001.

38. Neisendorfer, J., Localization and connected covers of finite complexes, in The Čech Centennial, Contemp. Math. 181, Boston, MA, 1993, pp. 385-390, Amer. Math. Soc., Providence, RI, 1995.

39. Porter, G. J., Homotopical nilpotence of $S^{3}$, Proc. Amer. Math. Soc. 15 (1964), 681-682.

40. RAO, V. K., Homotopy nilpotent Lie groups have no torsion in homology, Manuscripta Math. 92 (1997), 455-462.

41. Rector, D. L., Subgroups of finite dimensional topological groups, J. Pure Appl. Algebra 1 (1971), 253-273.

42. Rotman, J. J., An Introduction to the Theory of Groups, 4th ed., Graduate Texts in Mathematics 148, Springer, New York, 1995, xvi+513 pp. 
Cristina Costoya

UDC Computación

Edificio Área Científica, 3.04

ES-15071 A Coruña

Spain

cristina.costoya@udc.es

Jérôme Scherer

EPFL SB MATHGEOM

Station 8, MA B3 455

CH-1015 Lausanne

Switzerland

jerome.scherer@epfl.ch

Received May 25, 2016

in revised form May 29, 2017

\author{
Antonio Viruel \\ UMA Álgebra, Geometría y \\ Topología \\ Campus Teatinos \\ ES-29071 Málaga \\ Spain \\ viruel@uma.es
}

